\title{
Composição florística e variação sazonal da biomassa de macrófitas aquáticas em lagoa de meandro do Pantanal ${ }^{1}$
}

Floristic composition and seasonal variation in biomass of macrophytes in a meander lake in the Pantanal

\author{
Deborah Christiane Leite Kufner ${ }^{2,3}$,Edna Scremin-Dias ${ }^{2}$ \& Adriana Guglieri-Caporal ${ }^{2}$
}

\begin{abstract}
Resumo
Neste estudo é descrita a composição florística e analisada a influência do pulso de inundação sobre a biomassa de quatro espécies de macrófitas aquáticas, Eichhornia azurea, E. crassipes, Pistia stratiotes e Salvinia auriculata, ao longo de um ciclo hidrológico, em uma lagoa de meandro no Pantanal Mato Grosso do Sul. Foram identificados 42 táxons, inseridos em 34 gêneros e 25 famílias botânicas. As maiores riquezas foram obtidas para as famílias Araceae, Onagraceae e Poaceae, com quatro espécies cada, 14 famílias apresentaram apenas uma espécie, e o gênero mais representativo foi Ludwigia, com quatro espécies. Das formas biológicas, a que foi representada pelo maior número de táxons foi a emergente, seguida da anfibia e flutuante livre. As espécies analisadas apresentaram diferenças significativas ao longo do período sazonal nas duas regiões de amostragem $(\mathrm{p}<0,05)$. Mesmo não apresentando padrão na variação temporal da biomassa de todas as espécies estudadas, houve relação da biomassa de $E$. azurea e $E$. crassipes à flutuação do nível da água da lagoa.
\end{abstract}

Palavras-chave: biomassa, conectividade, planície de inundação, riqueza de espécies, sazonalidade.

\begin{abstract}
This study aims to describe the floristic composition and analyze the influence of flood pulse on the biomass of four species of aquatic macrophytes, Eichhornia azurea, E. crassipes, Pistia stratiotes and Salvinia auriculata, and during a hydrological cycle in a meander lake in the Brazilian Pantanal. We identified 42 taxa, 34 genera and 25 families. The richest families were Araceae, Onagraceae and Poaceae with four species each; 14 families had only one species. Ludwigia was the most representative genus (four species). The life forms that were represented by the greatest number of taxa were emergent, followed by amphibious and free-floating. Biomass of all species showed significant differences during the study period in the two sampling regions ( $\mathrm{p}<$ $0.05)$. Although not presenting a pattern in temporal biomass variation of all species, the biomass of both $E$. azurea and E. crassipes was related to fluctuating water levels of the lake.
\end{abstract}

Key words: biomass, connectivity, floodplain, species richness, seasonality.

\section{Introdução}

As planícies de inundação estão entre os ecossistemas mais produtivos do mundo (Neiff $e t$ al. 2006), no qual macrófitas aquáticas têm papel chave na estrutura e funcionamento (BarratSegretain et al. 1998), além de contribuírem de forma significativa para elevada biodiversidade desses ambientes, fator regulado principalmente pela variação da frequência e amplitude do nível da água (Ward \& Tockner 2001).

No Pantanal ocorrem diversos ambientes propícios ao estabelecimento e proliferação das macrófitas aquáticas, a exemplo dos corixos (curso d'água estacional), vazantes (canal de drenagem raso e amplo), baías (lagoas), caixas de empréstimo (corpo d'água feito para construção de estradas e/ ou tanques para gado que enchem de água periodicamente) e campos inundáveis (Pott \& Pott 2000), além de meandros abandonados, que se formam comumente em regiões de solo arenoso. A vegetação associada a esses locais compreende não apenas as plantas hidrófitas propriamente ditas, mas também as anfíbias, que periodicamente estão submersas ou margeiam estes ambientes (Bove et al. 2003).

\footnotetext{
${ }^{1}$ Parte da dissertação de Mestrado da primeira autora, Programa de Pós-Graduação em Biologia Vegetal, Universidade Federal de Mato Grosso do Sul

${ }^{2}$ Universidade Federal de Mato Grosso do Sul, Programa de Pós-Graduação em Biologia Vegetal, Cidade Universitária s/n ${ }^{\circ}$, CP. 549, 79070-900, Campo Grande, MS.

${ }^{3}$ Autor para correspondência: deborah.clk@gmail.com
} 
Além de cumprirem juntamente com as microalgas o papel de produtoras primárias e participarem da ciclagem e estocagem de nutrientes, as macrófitas contribuem para formação de detritos orgânicos e controle da poluição e eutrofização artificial (Esteves \& Camargo 1986; Pott \& Pott 2000), constituindo a principal comunidade produtora de biomassa no ecossistema aquático (Neiff 2000).

Em zonas temperadas, cujas estações climáticas são bem definidas, a dinâmica da produtividade das macrófitas aquáticas reflete alteração da biomassa no decorrer do ano; porém em regiões tropicais, devido à maior homogeneidade climática, há pequena variação deste parâmetro (Esteves 1998). Nesses locais os períodos de chuva e seca, bem como as flutuações do nível da água, são fatores determinantes na variação da biomassa (Wetzel 1975; Junk et al. 1989; Thomaz \& Bini 1998).

Muito embora aproximadamente $6,5 \%$ do território brasileiro sejam cobertos por áreas alagáveis, Pompêo \& Moschini-Carlos (2003) ressaltaram que o número de trabalhos publicados sobre macrófitas aquáticas no Brasil é muito baixo e a maioria são realizados em reservatórios. As áreas úmidas de todo o planeta estão fortemente ameaçadas e grande parte já foram destruídas (Meirelles et al. 2004). Segundo os autores essas perturbações podem ser evidenciadas por meio de mudanças florísticas e fitossociológicas em comparação com áreas similares preservadas.

No Pantanal há crescente impacto da atividade pesqueira e turística, fato comprovado pelo acúmulo de lixo nos rios e lagoas marginais, e pela presença de espécies ruderais em áreas úmidas. A flora é importante indicadora das condições ambientais de uma região, tanto no que se refere à proteção do solo, quanto na definição de hábitats para animais silvestres (Abdon et al. 1998). Apesar das listagens de espécies de determinada região serem muitas vezes consideradas como informações pouco apropriadas para leitura, é a forma mais prática de caracterizar a diversidade de espécies de determinada área (Pott 1999). Esse conhecimento é imprescindível para preservação e manejo de populações, já que qualquer alteração na sua dinâmica pode gerar respostas não desejadas, influenciando no desenvolvimento de espécies oportunistas, necessitando muitas vezes de manejo para o controle (Pompêo 2008), a fim de garantir a efetiva preservação da saúde ambiental.

Os meandros formados nos rios do Pantanal podem estar ativos, total ou parcialmente abandonados, influenciados pelo fluxo hídrico dos rios que lhe deram origem. A conectividade dessas formações, em maior ou menor grau, resulta em diferentes escalas de influência do nível da água sobre a biota ali estabelecida. Estudos em meandros envolvendo a avaliação da composição florística e quantificação de biomassa em resposta a variação temporal do nível das águas ainda são escassos. Neste estudo é descrita a composição florística e analisada a influência do pulso de inundação sobre a biomassa de quatro espécies de macrófitas aquáticas, Eichhornia azurea, E. crassipes, Pistia stratiotes e Salvinia auriculata, ao longo de um ciclo hidrológico, em uma lagoa de meandro no Pantanal Mato Grosso do Sul.

\section{Material e Métodos}

A área de estudo tem influência do rio Miranda, e localiza-se próximo à estrada Parque, sob coordenadas $19^{\circ} 30^{\prime} 36,97^{\prime \prime} \mathrm{S}$ e $57^{\circ} 06^{\prime} 9,53^{\prime}$ 'W na sub-região conhecida como Miranda-Abobral, município de Corumbá, Mato Grosso do Sul. Localmente, a lagoa de meandro é conhecida como Baía Negra, possui cerca de $3 \mathrm{~km}$ de extensão, em formato de "ferradura", com um de seus limites conectado ao rio durante o período de cheia.

O clima do Pantanal é tropical sub-úmido do tipo Aw, segundo Köppen (1948), com média pluviométrica de $1.110 \mathrm{~mm}$ e temperatura média de $26^{\circ} \mathrm{C}$ anual. É caracterizado por possuir duas estações bem definidas, uma chuvosa, que se estende de outubro a março, e outra relativamente seca, de abril a setembro (Wilhelmy 1957).

\section{Florística}

As coletas foram aleatórias abrangendo todas as comunidades existentes ao longo do perímetro do meandro, da periferia ao centro, durante cinco visitas, ao longo de um ciclo de inundação. Este procedimento visou relacionar como a presença das espécies do local variou com a disponibilidade hídrica, comparando os períodos de cheia (fevereiro e junho/2008) e de seca (novembro/2007, agosto e outubro/2008).

Para identificação taxonômica foi utilizada literatura específica, e quando necessário, comparação com material de herbário e consulta a especialistas. O material fértil foi depositado no Herbário CGMS da Universidade Federal de Mato Grosso do Sul.

A identificação das famílias de fanerógamas foi baseada no sistema de classificação do APG III - Angiosperm Phylogeny Group (2009), para as pteridófitas seguiu-se Kramer \& Green (1990), e para as briófitas Clarke \& Duckett (1979). A abreviação dos nomes de autores foi feita por consulta ao 
Brummitt \& Powell (1992) e as formas biológicas das macrófitas aquáticas foi baseada no proposto por Irgang et al. (1984).

\section{Biomassa}

A análise de biomassa foi realizada nas duas extremidades do meandro - ponto 1 (P1) e ponto 2 (P2) (Fig. 1), sendo realizada para as cinco espécies mais abundantes (avaliação visual): Eichhornia crassipes (Mart.) Solms, Pistia stratiotes L. e Salvinia auriculata Aubl. em P1, e E. azurea (Sw.) Kunth, $P$. stratiotes e $S$. auriculata em P2. Para cada ponto, a transparência e profundidade foram aferidas com disco de Sechhi e corda graduada.

Para estimar a biomassa, utilizou-se como amostrador um quadrado de $0,25 \mathrm{~m}^{2}$ (Westlake 1975), lançado aleatoriamente cinco vezes em cada ponto amostral. O material vegetal contido dentro do quadrado foi coletado, levado ao laboratório e lavado em água corrente para remoção de qualquer material aderido. Após a secagem em estufa a $65^{\circ} \mathrm{C}$ até atingir peso seco (PS) constante, a biomassa de cada espécie foi expressa em $\mathrm{gPS} \mathrm{m}^{-2}$.

Para verificar se houve diferença significativa na biomassa entre os períodos amostrais, foi realizado ANOVA e teste Tukey com 5\% de significância.

\section{Resultados e Discussão}

\section{Florística}

Foram catalogadas 42 espécies, distribuídas em 34 gêneros e 25 famílias entre briófitas, pteridófitas e fanerógamas (Tab. 1). O número de espécies obtido neste trabalho é similar ao relatado por Pott et al. (1989) em lagoas permanentes no Pantanal nas sub-regiões de Abobral e Nhecolândia. As famílias mais ricas foram Araceae, Onagraceae e Poaceae com quatro espécies cada (29\%), Asteraceae e Polygonaceae com três e as demais apresentaram duas espécies cada (Fig. 2). Do total de famílias presentes na lagoa de meandro, 14 apresentaram apenas uma espécie, cerca de 33\% das espécies catalogadas. Famílias que apresentaram mais de uma espécie, a exemplo de Cyperaceae, Fabaceae, Onagraceae e Poaceae, também foram citadas por outros autores como as mais abundantes e importantes em comunidades hidrófilas do Brasil (Irgang et al. 1984; Bove et al. 2003; Thomaz et al. 2004; Henry-Silva et al. 2010).

Em outras áreas úmidas do Pantanal, tanto com grande extensão de área marginal parcialmente alagada ao longo do ano ou em época chuvosa, como corpos d'água com pouca profundidade, também é citada a predominância da família Poaceae, como em Poconé (Nunes da Cunha \& Vilhalva 1999) e Aquidauana (Rocha et al. 2007). Essa predominância também foi verificada próxima a açudes no estado da Bahia (França et al. 2003) e em lagos inventariados do Amapá (Costa Neto et al. 2007).

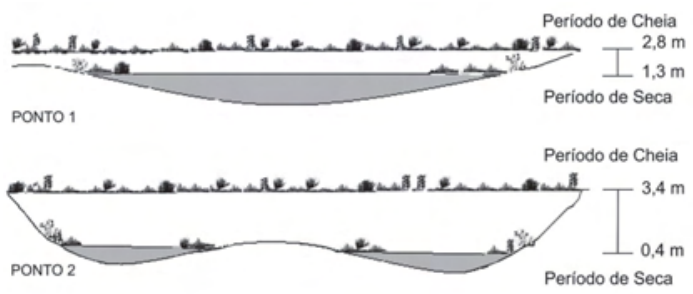

Figura 1 - Perfis esquemáticos dos dois pontos de amostragem (P1 e P2) na lagoa de meandro Baía Negra, Pantanal, sub-região de Miranda, evidenciando a cobertura de macrófitas aquáticas nos períodos de seca e cheia. Figure 1 - Schematic profiles of the two sampling points (P1 and P2) in the lake meander Baía Negra, Pantanal, sub-region of Miranda, showing the aquatic macrophyte cover during periods of drought and flood.

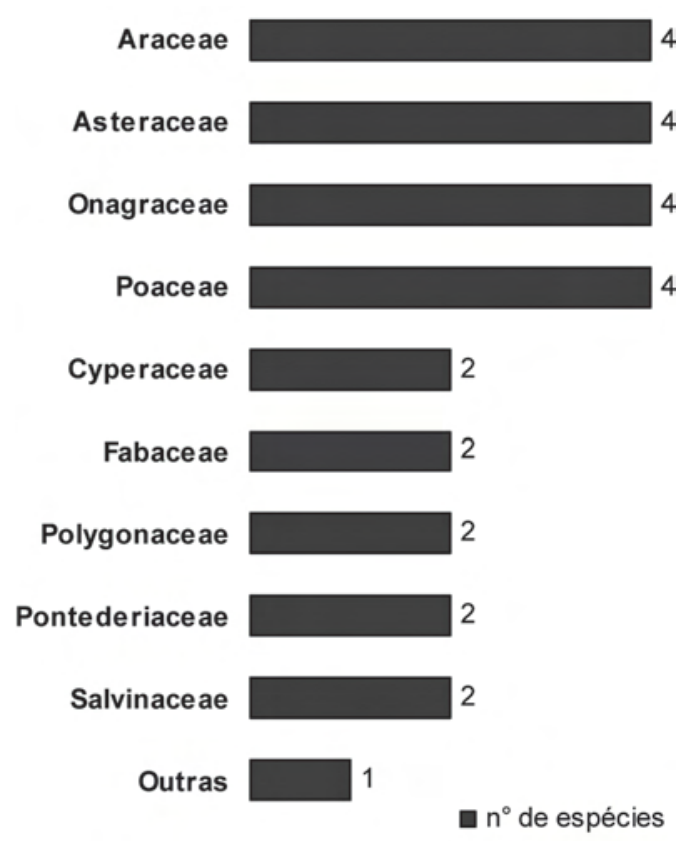

Figura 2 - Famílias botânicas mais representativas na lagoa Baía Negra no Pantanal, sub-região de Miranda. Figure 2 - Most representative botanical families in the lake meander Baía Negra, Pantanal sub-region of Miranda. 
Tabela 1 - Espécies vegetais coletadas na Baía Negra, Pantanal, sub-região de Miranda, seguidos do nome popular e forma biológica $(\mathrm{FB}): \mathrm{A}=$ anfíbia, $\mathrm{Ep}=$ epífita, $\mathrm{E}=$ emergente, $\mathrm{FF}=$ flutuante fixa, $\mathrm{FL}=$ flutuante livre $\mathrm{e} *=$ ruderal ou invasora.

Table 1 - Plant species collected Baía Negra, Pantanal, sub-region of Miranda. followed by the popular name and organic form (FB): $\mathrm{A}=$ amphibian, $\mathrm{Ep}=$ epiphyte, $\mathrm{E}=$ emergent, $\mathrm{FF}=$ Floating fixed and free floating $\mathrm{FL}=*$ = ruderal or weedy.

\begin{tabular}{llc}
\hline FAMÍLIA/Espécie & Nome popular & FB \\
\hline
\end{tabular}

\section{Briófita}

RICCIACEAE

Ricciocarpus natans (L.) Corda

Pteridófitas

AZOLLACEAE

Azolla filiculoides Lam.

PARKERIACEAE

Ceratopteris pteridoides (Hook.) Hieron.

SALVINIACEAE

Salvinia auriculata Aubl. *

Salvinia biloba Raddi

pé-de-sapo

orelha-de-onça

orelha-de-onça

chapéu-de-couro

E

Echinodorus paniculatus Micheli

ARACEAE

Lemna minuta Kunth

Pistia stratiotes L. *

Wolffiella lingulata (Hegelm.) Hegelm.

Wolffiella oblonga (Phil.) Hegelm.

ARALIACEAE

Hydrocotyle ranunculoides $\mathrm{L}$. $\mathrm{f}$.

ASTERACEAE

Aspilia latissima Malme

Enydra radicans (Willd.) Lack

Mikania micrantha Kunth

COMMELINACEAE

Commelina schomburgkiana Klotzsch

CONVOLVULACEAE

Ipomoea setifera Poir

CYPERACEAE

Cyperus gardneri Nees

Oxycaryum cubense (Poepp. \& Kunth) Palla

EUPHORBIACEAE

Caperonia castaneifolia (L.) A. St.-Hil.

FABACEAE

Calopogonium caeruleum (Benth.) Sauvalle

Vigna longifolia (Benth.) Verdc.

HELICONIACEAE

Heliconia marginata (Griggs) Pittier

HYDROCHARITACEAE

Limnobium laevigatum (Humb. \& Bonpl. ex Willd.) Heine alface d'água

FL

FL

FL

FL

chapéu-de-sapo

FF

mirassol

A

jasmin-do-brejo

A

A, E

santa-luzia

E

cipó-leiteiro

A

baceiro

Ep

baceiro

Ep

erva-de-bicho-branca

A, E

E

feijãozinho

E

pacova

A

camalotinho
FL

FL

FL

政

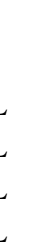

F

E




\begin{tabular}{|c|c|c|}
\hline FAMÍLIA/Espécie & Nome popular & FB \\
\hline \multicolumn{3}{|l|}{ MALVACEAE } \\
\hline Byttneria filipes Mart. ex K. Schum. & espinheiro & A \\
\hline Pavonia laetevirens R.E. Fr. & algodão-bravo & $\mathrm{E}, \mathrm{A}$ \\
\hline \multicolumn{3}{|l|}{ ONAGRACEAE } \\
\hline Ludwigia elegans (Cambess.) H. Hara & florzeiro & $\mathrm{E}$ \\
\hline Ludwigia helminthorrhiza (Mart.) H. Hara & lombrigueira & A,FF, FL \\
\hline Ludwigia leptocarpa (Nutt.) H. Hara & cruz-de-malta & $\mathrm{A}, \mathrm{E}$ \\
\hline Ludwigia octovalvis (Jacq.) P.H. Raven & & $\mathrm{A}, \mathrm{E}$ \\
\hline \multicolumn{3}{|l|}{ PHYLLANTHACEAE } \\
\hline Phyllanthus fluitans Benth. ex Müll. Arg. & orelha-de-onça & FL \\
\hline \multicolumn{3}{|l|}{ PLANTAGINACEAE } \\
\hline Angelonia salicariaefolia Humb. \& Bonpl. & & A \\
\hline \multicolumn{3}{|l|}{ POACEAE } \\
\hline Hymenachne donacifolia (Raddi) Chase & & E \\
\hline Panicum elephantipes Nees ex Trin. & capim-camalote & $\mathrm{E}, \mathrm{FF}$ \\
\hline Panicum mertensii Roth & felpudo & $\mathrm{E}$ \\
\hline Steinchisma laxum (Sw.) Zuloaga & grama-do-carandazal & $\mathrm{E}$ \\
\hline \multicolumn{3}{|l|}{ POLYGONACEAE } \\
\hline Polygonum acuminatum Kunth * & erva-de-bicho & $\mathrm{E}$ \\
\hline Polygonum ferrugineum Wedd. & erva-de-bicho & E \\
\hline Polygonum hispidum Kunth & erva-de-bicho & $\mathrm{E}$ \\
\hline \multicolumn{3}{|l|}{ PONTEDERIACEAE } \\
\hline Eichhornia azurea (Sw.) Kunth & camalote & FF \\
\hline Eichhornia crassipes (Mart.) Solms & camalote & $\mathrm{A}, \mathrm{FL}$ \\
\hline \multicolumn{3}{|l|}{ RUBIACEAE } \\
\hline Staelia thymbroides (Mart. ex Mart. \& Zucc.) K. Schum & & A \\
\hline \multicolumn{3}{|l|}{ VERBENACEAE } \\
\hline Lippia alba (Mill.) N.E.Br. ex Britton \& P.Wilson & cidreira-do-campo sálvia & A \\
\hline
\end{tabular}

$\mathrm{O}$ gênero de maior riqueza foi Ludwigia L. (Onagraceae), com quatro espécies $(9,5 \%)$, seguido por Polygonum L. (Polygonaceae) com três $(7,1 \%)$, e por Eichhornia Kunth (Pontederiaceae), Panicum L. (Poaceae), Salvinia Ség. (Salvinaceae) e Wolffiella Hegelm. (Araceae), com duas espécies cada, representando juntas $19 \%$ do número total de espécies. O gênero Ludwigia também foi citado por Pivari et al. (2008) como o mais representativo para baceiros (ilhas de macrófitas aquáticas flutuantes) no Pantanal das sub-regiões do Abobral e Miranda. Tal riqueza pode ser explicada pela presença de espécies desse gênero em todos os estádios de sucessão, resultando na capacidade destas espécies explorarem diferentes ambientes (Pott $\&$ Pott 2000), além de apresentarem muitas espécies anfíbias, aumentando a amplitude de distribuição de espécies deste gênero nas áreas úmidas.
A forma biológica emergente predominou com 17 espécies, seguida das anfíbias com 14 e flutuantes livres com 13 (Tab. 1). Considerando que as coletas também foram realizadas no entorno da lagoa, a distribuição dessa forma biológica era esperada, já que as espécies que habitam estas áreas possuem adaptações morfológicas, anatômicas e ecofisiológicas às variações do nível da água geradas pela sazonalidade (Cronk \& Fennessy 2001; Scremin-Dias 2000, 2009).

Os dados de florística revelam que o nível da água pode regular a frequência e a ocorrência de algumas espécies e que estas não são excluídas, mas favorecidas em determinados períodos sazonais. Não foram encontradas espécies submersas, provavelmente devido à turbidez da água que impossibilita o desenvolvimento desse grupo de plantas. 
A ocorrência de espécies ruderais ou invasoras $(7 \%)$ evidencia certo estado de antropização. Embora, no momento esta situação não pareça preocupante, práticas agropecuárias comuns na região podem intensificar e desencadear processo irreversível de alteração da flora original.

\section{Biomassa}

Diferenças significativas na biomassa das espécies Eichhornia crassipes, Pistia stratiotes e Salvinia auriculata para o Ponto 1 (P1), e E. azurea para o Ponto 2 (P2), foram obtidas para as duas áreas do meandro ao longo do período amostral (Fig. 3). A variação hidrológica na lagoa aparentemente atuou de maneira desigual sobre a biomassa das espécies estudadas. Isso pode ser explicado pelas diferenças na competição por luz, nutrientes e espaço, de modo que as maiores taxas de crescimento não ocorrem simultaneamente em diferentes espécies que coexistem em um mesmo ecossistema aquático (Neiff 2000).

O meandro apresentou valor máximo de profundidade de 5,30 $\mathrm{m}$ na região central, e o mínimo de $0,40 \mathrm{mem}$ P2 (Fig. 1), com o nível da água atingindo de 2,8 a 3,4 m no pico da cheia e na seca reduziu para 1,3 e $0,4 \mathrm{~m}$, nos $\mathrm{P} 1$ e $\mathrm{P} 2$ respectivamente.

Comparando-se os períodos de cheia e seca, os valores médios obtidos para a biomassa das espécies avaliadas, no ponto P1, Eichhornia crassipes apresentou $280,8 \mathrm{gPS} \mathrm{m}^{-2}$ na cheia a $12,1 \mathrm{gPS} \mathrm{m}^{-2}$ na seca; Pistia stratiotes variou de $4 \mathrm{~g} \mathrm{PS} \mathrm{m}^{-2}$ na cheia a $25,1 \mathrm{gPS} \mathrm{m}^{-2}$ na seca, e Salvinia auriculata teve maior valor na cheia, $22,4 \mathrm{gPS} \mathrm{m}^{-2}$, reduzindo para $6,5 \mathrm{gPS} \mathrm{m}^{-2}$ na seca.

Para o P2 os valores de biomassa de $E$. azurea, variaram de 741,6 $\mathrm{gPS} \mathrm{m}^{-2}$ na cheia a 397,5 $\mathrm{gPS} \mathrm{m}^{-2}$ na seca. $P$. stratiotes teve maior biomassa na estação cheia, com 39,2 gPS m${ }^{-2}$ e a menor na seca, $11 \mathrm{gPS} \mathrm{m}^{-2}$. O contrário ocorreu para $S$. auriculata, cujos valores de biomassa foram menores na cheia, $6 \mathrm{gPS} \mathrm{m}^{-2}$ do que na seca, $8,9 \mathrm{gPS}$ $\mathrm{m}^{-2}$, demonstrando nenhuma relação entre o nível d'água e a biomassa para essas duas últimas espécies.

Aqui, junto à diminuição do nível da água, as populações de E. azurea e E. crassipes reduziram em ambas áreas amostradas. Nesse período, houve o aparecimento de bancos de areia em P2, resultando no recuo e redução gradual das macrófitas, que se ancoraram às margens da lagoa (Fig. 1). Este estudo confirma os dados obtidos por Bini (1996) no Paraná para E. azurea, Pedralli (2003) em Minas Gerais para E. crassipes e Da Silva \& Esteves (1993) no Pantanal para as mesmas espécies. Em ambos houve aumento da biomassa dessas espécies no período de cheia. Padrões diferentes foram observados para $E$. azurea por Henry-Silva \& Camargo (2003) em um rio de São Paulo, segundo os autores provavelmente em virtude da constância hidrológica local.

De acordo com Ludlow (1987), juntamente com as alterações do nível da água, a temperatura também pode influenciar a produtividade das macrófitas, levando as espécies a apresentar ajustes morfológicos e de produção de biomassa.Resultados obtidos neste estudo para E. crassipes em P1 corroboram Knipling et al. (1970) onde os mesmos relataram que altas temperaturas estimulam o ganho de biomassa dessa espécie que aproveitam de forma eficiente a energia solar quando a população encontra-se adensada.

A elevação da coluna d'água resultou em alta taxa de mortalidade de E. crassipes em P1, com redução de 23 vezes do valor da biomassa em comparação com a época seca. Esta espécie, flutuante livre, possui seu sistema radicular distribuído na coluna d'água, onde os nutrientes encontram-se então mais diluídos (Scremin-Dias 2000, 2009). Em contrapartida, E. azurea apresentou aumento nos valores de biomassa após o distúrbio causado pela repentina elevação da coluna d'água. Com seus indivíduos enraizados no substrato e folhas flutuantes, essa espécie parece estar mais adaptada a essa dinâmica hidrológica. $\mathrm{O}$ acesso aos nutrientes do solo, devido ao enraizamento, associado ao crescimento clonal e potencial de fragmentação permite um desenvolvimento efetivo, possibilitando a esta espécie alcançar elevados valores de biomassa (Westlake 1963).

Quando em baixas densidades, Pistia stratiotes cobre o espelho d'água rapidamente, uma vez que investe em propagação vegetativa sob essas condições (Coelho et al. 2005a). Provavelmente essa seria uma das estratégias de resistência às condições adversas, sugerindo que no período de seca em P2, essa espécie invista nesse tipo de reprodução, assegurando que seus diásporos permaneçam na área durante o período de menor disponibilidade hídrica e, que na época cheia, cresçam e restabeleçam a população no local.

$\mathrm{O}$ ponto $\mathrm{P} 1$ apresentou mais biomassa por área que em P2, provavelmente devido à diferença morfológica entre as espécies de Pontederiaceae. Em P2, E. azurea apresenta ampla propagação vegetativa, com estolões crescendo em ziguezague entre os limbos, permitindo incidência solar na 

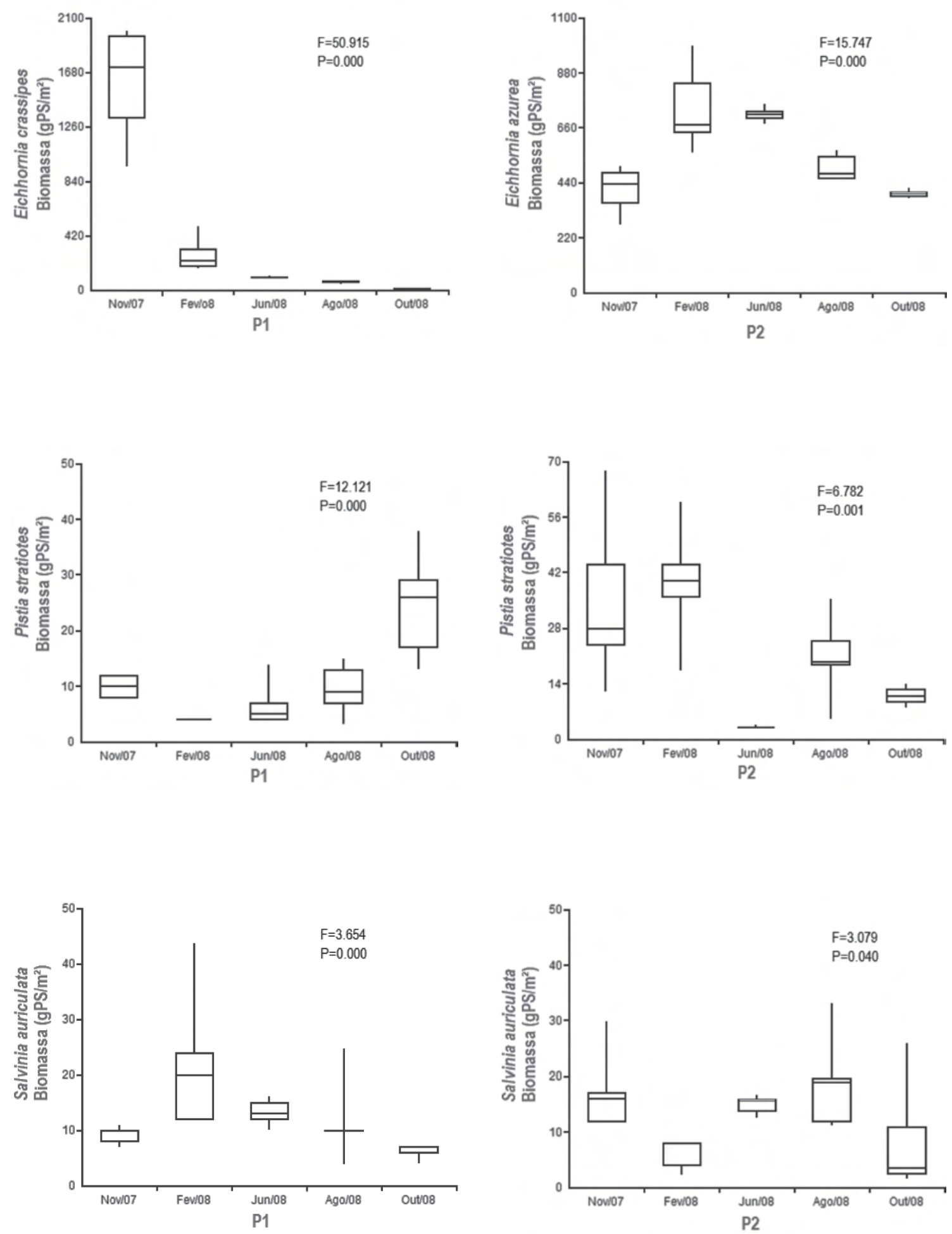

Figura 3 - Variação sazonal da biomassa das macrófitas aquáticas estudadas na lagoa Baía Negra no Pantanal, subregião de Miranda.

Figure 3 - Seasonal variation of biomass of aquatic macrophytes in the lake meander Baía Negra, Pantanal, sub-region of Miranda. 
superfície da água, impulsionando o desenvolvimento de $P$. stratiotes. Já em P1, a espécie E. crassipes apresentou crescimento vertical acima da lâmina d'água intenso, principalmente quando estocada em altas densidades, sendo aparentemente mais favorecida pela energia solar que $P$. stratiotes. Esta pode ter tido seu desenvolvimento afetado pelo sombreamento produzido por E. crassipes, levando à redução de sua biomassa durante o período da cheia.

Os diferentes resultados obtidos por Pistia stratiotes e Salvinia auriculata nos dois pontos de amostragem revelaram que não há um padrão específico de produção de biomassa em relação ao nível da água. Provavelmente essas espécies competem por um mesmo recurso, variável entre os pontos de amostragem, explicando assim o aumento de biomassa de uma e redução de outra para mesmo período, em pontos diferentes. Além disso, o teor de matéria orgânica disponível nos dois pontos em decorrência da decomposição da necromassa pode favorecer o desenvolvimento das espécies.

É interessante notar que espécies de macrófitas respondem diferentemente aos estímulos ambientais, quanto à produção de novas unidades vegetativas ou de reprodução sexuada. A espécie Salvinia auriculata produz maior proporção de esporocarpos sob alta densidade populacional, investindo em propagação vegetativa quando em baixas densidades (Coelho et al. 2005b). A alta produção de esporos é uma estratégia de sobrevivência ao eventual período de seca, já que o aumento da densidade pode ser um indicativo de que o nível da água está baixando, e os esporos consistem em estrutura de resistência à dessecação (Hoffman \& Stockey 1994).

Em regiões com alagamentos periódicos como o Pantanal, a propagação vegetativa pode assegurar a rápida colonização das espécies enquanto o nível d'água sobe e o crescimento de clones se torna possível (Hoffman \& Stockey 1994), o que é corroborado pelo aumento da produção de biomassa de $S$. auriculata obtido no presente estudo para o mesmo período. Apesar de ter havido grande variação do nível da água, não houve exclusão total da água nos dois pontos inventariados. Sendo assim, a diminuição dos indivíduos desta espécie nos pontos pode estar refletindo a convergência de vários fatores abióticos locais, difíceis de serem avaliados e interpretados pelo método empregado.

Devido à imprevisibilidade de eventos macroclimáticos, como cheias e secas, tem-se sugerido que a capacidade dos modelos de avaliação da produção de biomassa por macrófitas aquáticas, em longos períodos de tempo, ainda é baixa (Carr $e t$ al. 1997). Santos (2004) também cita a dificuldade em descrever matematicamente os vários fatores que podem influenciar positiva ou negativamente a produção de biomassa por esta forma de vida. Essa dificuldade é demonstrada pelos resultados aqui obtidos, onde a resposta ao pulso de inundação não foi padrão para todas as espécies avaliadas. Isto indica que a sazonalidade hídrica é apenas um dos vários dos fatores influentes e que estudos mais abrangentes são necessários para entender as respostas intra e interespecíficas das macrófitas aquáticas à inundação nas planícies alagáveis do Brasil Central.

\section{Agradecimentos}

A primeira autora agradece à Fundect/CAPES, a bolsa de Mestrado concedida. A terceira autora agradece a CAPES e a bolsa Prodoc concedida entre 2006 e 2009. A Antônio Fernando Monteiro Camargo pelas valiosas críticas e recomendações, e à Zildamara dos Reis Holsback Menegucci, a ajuda nas análises estatísticas.

\section{Referências}

Abdon, M.N.; Silva, J.S.; Pott, V.J.; Pott, A. \& Silva, M.P. 1998. Utilização de dados analógicos do Landsat-TM na discriminação da vegetação de parte da sub-região da Nhecolândia no Pantanal. Pesquisa Agropecuária Brasileira, Brasília 33: 1799-1813.

APG III. 2009. An update of the Angiosperm Phylogeny Group classification for the orders and families of flowering plants: APG III. Botanical Journal of the Linnean Society 161: 105-121.

Barrat-Segretain, M.H.; Bornette, G. \& Hering-VilasBôas, A. 1998. Comparative abilities of vegetative regeneration among aquatic plants growing in disturbed habitats. Aquatic Botany 60: 201-211.

Bini, L.M. 1996. Influence of flood pulse on the phytomass of three species of aquatic macrophytes in the upper River Parana floodplain. Arquivos de Biologia e Tecnologia 39: 715-721.

Bove, C.P.; Gil, A.S.B.; Moreira, C.B. \& Anjos, R.F.B. 2003. Hidrófitas fanerogâmicas de ecossistemas aquáticos temporários da planície costeira do estado do Rio de Janeiro, Brasil. Acta Botanica Brasilica 17: 119-135.

Brummitt, R.K. \& Powell, C.E. 1992. Authors of plant names. The Royal Botanic Gardens, Kew. 732p.

Carr, G.M.; Duthie, H.C. \& Taylor, W.D. 1997. Models of aquatic plant productivity: a review of the factors that influence growth. Aquatic Botany, Amsterdam. 59: 195-215. 
Clarke, G.C.S. \& Duckett, J.C. 1979. Bryophyta Systematics. Academic Press London. 572p.

Coelho, F.F.; Lopes, F.S. \& Sperber, C.F. 2005a. Persistance strategy of Salvinia auriculata Aublet in temporary ponds of Southern Pantanal, Brazil. Aquatic Botany 81: 343-352.

Coelho, F.F.; Deboni, L. \& Lopes, F.S. 2005b. Densitydependent reproductive and vegetative allocation in the aquatic plant Pistia stratiotes (Araceae). Revista de Biologia Tropical 53: 369-376.

Costa Neto, S.V.; Senna, C.; Tostes, L.C.L. \& Silva, S.R.M. 2007. Macrófitas aquáticas das Regiões dos Lagos do Amapá, Brasil. Revista Brasileira de Biociências 5: 618-620.

Cronk, J.K. \& Fennessy, M.S. 2001. Wetlands plants: biology and ecology. CRC Press, Boca Raton. 462p.

Da Silva, C.J. \& Esteves, F.A. 1993. Biomass of three macrophytes in the Pantanal of the Mato Grosso, Brazil. International Journal of Ecology and Environmental Sciences 19: 11-23.

Esteves, F.A. 1998. Fundamentos de limnologia. 2ed. Interciência/FINEP, Rio de Janeiro. 602p.

Esteves, F.A. \& Camargo, A.F.M. 1986. Sobre o papel das macrófitas na estocagem e ciclagem de nutrientes. Acta Limologica Brasilica 1: 273-298.

França, F.; Melo, E.; Goes Neto, A.; Araújo, D.; Bezerra, M.; Ramos, H.; Castro, I. \& Gomes, D. 2003. Flora vascular de açudes de uma região do semi-árido da Bahia, Brasil. Acta Botanica Brasilica 17: 549-559.

Henry-Silva, G.G. \& Camargo, A.F.M. 2003. Avaliação sazonal da biomassa da macrófita aquática Eichhornia azurea em um rio de águas brancas da bacia hidrográfica do rio Itanahém (litoral sul do Estado de São Paulo, Brasil). Hoehnea 30: 71-77.

Henry-Silva, G.G.; Moura, R.S.T. \& Dantas, L.L.O. 2010. Richness and distribuition of aquatic macrophytes in Brazilian semi-arid aquatic ecosystems. Acta Limnologica Brasiliensia 22: 147-156.

Hoffman, G.L. \& Stockey, R.A. 1994. Sporophytes, megaspores, and massules of Azolla stanleyi from the Paleocene Joffre Bridge locality, Alberta. Canadian Journal of Botany 72: 301-308.

Irgang, B.E.; Pedralli, G. \& Waechter, J.I. 1984. Macrófitas aquáticos da Estação Ecológica do Taim, Rio Grande do Sul, Brasil. Roessleria 6: 395-404.

Junk, W.J.; Bayley, P.B. \& Sparks, R.E. 1989. The flood pulse concept in river - floodplain systems. Canadian Special Publication of Fisheries and Aquatic Sciences 106: 110-127.

Knipling, E.D.; West, S.H. \& Haller, W.T. 1970. Growth characteristics, yield potential, and nutritive content of water hyacinth. Soil \& Crop Society 30: 51-63.

Köppen, W. 1948. Climatologia: con un estudio de los climas de la tierra. Fondo de Cultura Econômica, Ciudad de México. 479p.
Kramer, K.U. \& Green, P.S. 1990. Pteridophytes and Gymnosperms. Vol. I. In: Kubitzki, K. (ed.). The families and genera of vascular plants. SpringerVerlag, Berlin. 404p.

Ludlow M.M. 1987. Light stress at high temperature. In: Kylem D.J.; Osmondm C.B.; Arntzenm C.J. (eds.). Photoinhibition (9). 2ed. Elsevier Science Publishers, Amsterdam. Pp. 90-107.

Meirelles, M.L.; Guimarães, A.J.M.; Oliveira, R.C.; Araújo, G.M. \& Ribeiro, J.F. 2004. Impactos sobre o estrato herbáceo de áreas úmidas do Cerrado. In: Aguiar, L. M. S. \& Camargo, A. J. A. (org.). Cerrado: ecologia e caracterização. Vol. 1. Embrapa Cerrados, Planaltina. Pp. 41-68.

Neiff, J.J. 2000. Diversity in some tropical wetland systems of South America: 1-32. In: Gopal, B. \& Junk, W. (eds.). Wetlands biodiversity. Vol. 2. Backhuys Publish, Leiden. Pp. 31-60.

Neiff, A.P.; Neiff, J.J. \& Casco, S.L. 2006. Leaf litter decomposition in three wetland types of the Paraná River floodplain. Wetlands 26: 558-566.

Nunes da Cunha, C. E. \& Vilhalva, D. A. 1999. Lista preliminar de um campo inundável e brejo, Fazenda Retiro Novo, Pantanal de Poconé, MT. In: III Simpósio sobre Recursos Naturais e Socioeconômicos do Pantanal. EMBRAPA, Brasília.

Pedralli, G. 2003. Macrófitas aquáticas como bioindicadoras da qualidade da água: alternativas para usos múltiplos em reservatórios. In: Thomaz, S.M. \& Bini, L.M. (eds.) Ecologia e manejo de macrófitas aquáticas. EDUEM, Maringá. Pp. 171-188.

Pivari, M.O.; Pott, V.J. \& Pott, A. 2008. Macrófitas aquáticas de ilhas flutuantes (Baceiros) nas subregiões do Abobral e Miranda, Pantanal, MS, Brasil. Acta Botanica Brasilica 22: 563-571.

Pompêo, M. 2008. Monitoramento e manejo de macrófitas aquáticas. Ecol. Bras. 12: 406-424.

Pompêo, M.L.M. \& Moschini-Carlos, V. 2003. Macrófitas aquáticas e perifíton: aspectos ecológicos e metodológicos. Rima, São Carlos. 124p.

Pott, V.J. 1999. Riqueza verde em meio azul. In: ScreminDias, E.; Pott, V.J.; Hora, R.C. \& Souza, P.R. (eds.). Nos jardins submersos da Bodoquena: guia para identificação de plantas aquáticas de Bonito e região. Editora da UFMS, Campo Grande. 160p.

Pott, V.J.; Bueno, N.C.; Pereira, R.A.C.; Salis, S.M. \& Vieira, N.L. 1989. Distribuição de macrófitas aquáticas numa lagoa na fazenda Nhumirim, Nhecolândia, Pantanal, MS. Acta Botanica Brasilica (Supl.) 3: 153-168.

Pott, V.J. \& Pott, A. 2000. Plantas aquáticas do Pantanal. Embrapa, Brasília. 404p.

Rocha, C.G.; Resende, U.M. \& Lugnani, J.S. 2007. Diversidades de macrófitas em ambientes aquáticos do IPPAN na Fazenda Santa Emília, Aquidauana, MS. Revista Brasileira de Biociências 5: 456-458. 
Santos, A.M. 2004. Macrófitas aquáticas da planície de inundação do alto Rio Paraná: produtividade primária, decomposição, ciclagem de nutrientes e diversidade. Tese de Doutorado. Universidade de Maringá, Maringá. 101p.

Scremin-Dias, E. 2000. A plasticidade fenotípica das macrófitas aquáticas em resposta à dinâmica ambiental. In: Embrapa Recursos Genéticos e Biotecnologia. Tópicos especiais em botânica 1. Embrapa, Brasília. Pp. 84-90.

Scremin-Dias, E. 2009. Tropical aquatic plants: morphoanatomical adaptations. In: Del-Claro, K. \& Rico-Gray (orgs.). Encyclopedia of tropical biology and conservation management. Vol 1. UNESCO/EOLSS, Paris. Pp. 84-132.

Thomaz, S.M. \& Bini, L.M. 1998. Ecologia e manejo de macrófitas aquáticas em reservatórios. Acta Limnologica Brasiliensia 10: 103-116.

Thomaz, D.O.; Costa Neto, S.V. \& Tostes, L.C.L. 2004. Inventário florístico das ressacas das bacias do igarapé da Fortaleza e do rio Curiaú. In: Takiyama, L.R. \& Silva, A.Q. (orgs.). Diagnóstico de ressacas do estado do Amapá: bacias do igarapé da Fortaleza e do rio Curiaú. GEA/SETEC/IEPA, Macapá. Pp. 13-32.

Ward, J. \& Tockner, K. 2001. Biodiversity: towards a unifying theme for river ecology. Freshwater Biology 46: 807-819.

Westlake, D.F. 1963. Comparisons of plant productivity. Biological Reviews 38: 385-425.

Westlake, D.F. 1975. Macrophytes. In: Whitton, B.A. (ed.). River ecology. University of California Press, Berkeley. Pp. 106-128.

Wetzel, R.G. 1975. Limnology. Sauders, Philadelphia. $743 p$.

Wilhelmy, H. 1957. Das grosse Pantanal in Mato Grosso. Deutscher Geographentag, Wuerzburg. In: Tagunsberichte und wissenchaftlichen Abhandlungen. Franz Steiner, Wiesbaden. Pp. 45-71. 